\title{
5-Azacytidine-Induced Cardiomyocyte Differentiation of Very Small Embryonic-Like Stem Cells
}

\author{
XiaoLin Sun $\mathbb{D}^{1},{ }^{1}$ HongXiao Li $\mathbb{D},{ }^{2}$ Ye Zhu $\mathbb{D},{ }^{2}$ Pei Xu $\mathbb{D}^{\mathbb{D}},{ }^{3}$ QiSheng Zuo $\mathbb{D}^{4},{ }^{4}$ BiChun Li $\mathbb{D}^{4},{ }^{4}$ \\ and Xiang Gu iD ${ }^{1,2}$ \\ ${ }^{1}$ Institute of Translational Medicine, Medical College, Yangzhou University, Yangzhou 225001, China \\ ${ }^{2}$ Department of Cardiology, Northern Jiangsu People's Hospital, Yangzhou, Jiangsu 225001, China \\ ${ }^{3}$ Taizhou People's Hospital, Taizhou, Jiangsu 225300, China \\ ${ }^{4}$ College of Animal Science and Technology, Yangzhou University, Yangzhou, Jiangsu 225001, China
}

Correspondence should be addressed to Xiang Gu; guxiang@yzu.edu.cn

Received 17 February 2020; Revised 29 May 2020; Accepted 16 June 2020; Published 8 September 2020

Academic Editor: Stefan Arnhold

Copyright (C) 2020 XiaoLin Sun et al. This is an open access article distributed under the Creative Commons Attribution License, which permits unrestricted use, distribution, and reproduction in any medium, provided the original work is properly cited.

The use of stem cells in generating cell-based pacemaker therapies for bradyarrhythmia is currently being considered. Due to the propensity of stem cells to form tumors, as well as ethical issues surrounding their use, the seed cells used in cardiac biological pacemakers have limitations. Very small embryonic-like stem cells (VSELs) are a unique and rare adult stem cell population, which have the same structural, genetic, biochemical, and functional characteristics as embryonic stem cells without the ethical controversy. In this study, we investigated the ability of rat bone marrow- (BM-) derived VSELs to differentiate in vitro into cardiomyocytes by 5-Azacytidine (5-AzaC) treatment. The morphology of VSELs treated with $10 \mu \mathrm{M} 5$-AzaC increased in volume and gradually changed to cardiomyocyte-like morphology without massive cell death. Additionally, mRNA expression of the cardiomyocyte markers cardiac troponin-T ( $\mathrm{cTnT}$ ) and $\alpha$-sarcomeric actin ( $\alpha$-actin) was significantly upregulated after 5 AzaC treatment. Conversely, stem cell markers such as Nanog, Oct-4, and Sox2 were continuously downregulated posttreatment. On day 14 post-5-AzaC treatment, the positive expression rates of cTnT and $\alpha$-actin were $18.41 \pm 1.51 \%$ and $19.43 \pm 0.51 \%$, respectively. Taken together, our results showed that rat BM-VSELs have the ability to differentiate into cardiomyocytes in vitro. These findings suggest that VSELs would be useful as seed cells in exploring the mechanism of biological pacemaker activity.

\section{Introduction}

Bradyarrhythmias are a group of life-threatening diseases, including sinus syndrome and atrioventricular block, and are characterized by cardiac pacing or conduction dysfunction. Since the late 1950s, bradyarrhythmia therapy has focused on implantation of electronic pacemakers [1]. Although electronic pacemakers are effective, they have several drawbacks and limitations, including infection, metal allergies, electrode dislocation, electronic interference, and lack of neurohormone responsiveness. Biological pacemakers provide an alternative to bradyarrhythmia electronic pacemakers and are generated by somatic gene transfection, cell fusion, or cell transplantation. However, biological pacemakers have been slow to be phased in as a bradyarrhythmia treatment due to the uncertainty of gene transfection targeting and pacing efficiency. Although cellular biological pacemakers have many advantages, there have been difficulties selecting seed cells and induction methods.

Kucia et al. [2] first identified a class of cells in adult mice that maintained pluripotent stem cell activity with crossdermal differentiation. These cells, known as very small embryonic-like stem cells (VSELs), have been successfully isolated from various tissues from multiple species and contain different surface markers [3-6]. In vitro transplantation experiments have shown that VSELs can differentiate into 
three different embryo layers without forming teratomas following transplantation into the immunodeficient mice $[7,8]$. Therefore, VSELs could potentially function as new seed cells for in vitro differentiation into a cardiomyogenic, biological pacemaker cell lineage.

The development of a successful cardiomyogenic cell lineage depends on adequate cellular differentiation and proliferation. Cellular differentiation and proliferation are heavily influenced by DNA methylation, and high DNA methylation levels limit these processes. Chemical inhibition of DNA methyltransferases can be effective in overcoming cell growth limitations. 5-Azacytidine (5-AzaC) is a common DNA methyltransferase inhibitor. By covalently binding to DNA methyltransferase, 5-AzaC reduces DNA methyltransferase activity, thereby reducing the methylation level and inducing cell proliferation and differentiation. Several studies have shown that 5-AzaC could induce differentiation of different types of stem cells into cardiomyocytes $[4,8,9]$. Furthermore, these differentiated cells exhibit spontaneous pulsation and measurable action potentials, consistent with cardiomyocytes $[4,8,9]$.

In this study, we isolated VSELs from rat bone marrow (BM) and examined whether 5-AzaC treatment could successfully differentiate BM-VSELs into cardiomyocytes. The BM-VSELs retained stem cell characteristics, and treatment with 5-AzaC induced BM-VSEL differentiation into cardiomyocytes. These data provide novel insights into using VSELs and 5-AzaC treatments for building an effective, extracorporeal biological pacemaker.

\section{Materials and Methods}

2.1. Isolation and Culturing of BM-VSELs. To obtain a highpurity rat BM-derived population of VSELs, this study adopted the sorting scheme for VSELs proposed by LabedzMaslowska et al. [4]. All procedures involving the care and use of animals conformed to the Animal Research: Reporting of In Vivo Experiments (ARRIVE) guidelines and were approved by the laboratory animal management and experimental animal ethics committee of Yangzhou University.

Bone marrow from 8-12-week-old, male or female, Wistar rats was collected for isolation of VSELs. Rat tibias and femurs were removed and any remaining muscle or connective tissue discarded. The BM was rinsed with phosphatebuffered saline (PBS) (Solarbio, Beijing, China). Total nucleated cells were obtained by lysing red blood cells with $1 \mathrm{x} \mathrm{BD}$ Pharm Lyse Buffer (BD Pharmingen, San Jose, CA, USA) for $10 \mathrm{~min}$. The nucleated cells were incubated with anti-CD45, anti-CD106, and anti-Lin monoclonal antibodies (1:200) at room temperature for $30 \mathrm{~min}$ and then sorted by flow cytometry to obtain CD $45^{-} \mathrm{Lin}^{-} \mathrm{CD} 106^{+}$VSELs. The following anti-rat antibodies (BD Pharmingen) were used for staining: (i) anti-CD45 (Alexa Fluor 647, clone: OX-1), (ii) FITC-conjugated Lin markers (anti-TCR $\alpha \beta$ (clone: R73), anti-CD3 (clone: 1F4), anti-CD11b/c (clone: OX-42), and anti-CD45RA (clone: OX-33)), and (iii) anti-CD106 (PE, clone: MR-106).

VSELs were cultured in Dulbecco's Modified Eagle's Medium (DMEM) (Thermo Fisher Scientific, Waltham,
MA, USA) containing $10 \%$ fetal bovine serum (FBS) (Biowest, Nuaille, France) at $37^{\circ} \mathrm{C}$ in a $5 \% \mathrm{CO}_{2}$ incubator. During VSEL expansion and proliferation, culture medium was replaced every three days and cells were passaged after they reached $70 \%$ confluency.

2.2. Immunofluorescence Staining to Identify VSEL Characteristics. VSELs in healthy growth conditions were plated in 24-well plates with climbing slides. Adherent growth was detected after 24 hours by immunofluorescence staining. VSELs were fixed with $4 \%$ paraformaldehyde at $4^{\circ} \mathrm{C}$ for $30 \mathrm{~min}$. Fixed cells were permeabilized with $0.1 \%$ Triton X-100 (Solarbio) and blocked with blocking solution (2\% FBS/0.1\% Tween 20 (Solarbio)). Fixed cells were incubated overnight at $4{ }^{\circ} \mathrm{C}$ with primary antibodies $(1: 200$ dilution) against rat Nanog, Oct-4, and Sox 2 (Proteintech, Chicago, IL, USA). Cells stained with diluent only served as the negative control. After overnight incubation, cells were washed with PBS three times for 5 min each, incubated with CY3-conjugated secondary antibody (1:500; Beyotime, Shanghai, China) for 1 hour at room temperature, and washed five times with PBS. Nuclei were stained with $4^{\prime}, 6$ diamidino-2-phenylindole (Solarbio). Slides were mounted and examined using a fluorescent microscope (TE2000 Nikon, Tokyo, Japan).

\subsection{Identification of VSELs by Alkaline Phosphatase Staining.} VSELs in healthy growth conditions were plated in 24-well plates with climbing slides, further stained with alkaline phosphatase (AKP) (Solarbio) staining solution, and observed with an inverted microscope (DM1750M Leica, Weztlar, Germany).

2.4. Treatment of VSELs with 5-AzaC. VSELs were divided into two separate groups: (1) untreated controls and (2) VSELs treated with various concentrations $(1 \mu \mathrm{M}, 10 \mu \mathrm{M}$, or $30 \mu \mathrm{M}$ ) of 5 -AzaC (Sigma-Aldrich Co., St. Louis, MO, USA). After overnight incubation, the medium was aspirated and cells were washed with PBS three times for 3-5 min. New culture medium was added and subsequently replaced every three days. After 5-AzaC treatment, cell morphology was observed daily using a phase contrast microscope (DMILPH1 Leica).

2.5. Cell Viability Assay. VSELs were seeded into 96-well plates at 5,000 cells per well. Cells were stained with $100 \mu \mathrm{L}$ sterile MTT dye $\left(0.5 \mathrm{mg} / \mathrm{mL}\right.$, Solarbio) for 4 hours at $37^{\circ} \mathrm{C}$ on days $0,1,7,14$, and 21 postseeding. Formazan crystals were solubilized with $100 \mu \mathrm{L}$ DMSO (Solarbio). Absorbance was measured at $490 \mathrm{~nm}$ using an automatic microplate reader (Multiskan ${ }^{\mathrm{TM}}$ FC, Thermo Fisher Scientific).

2.6. Flow Cytometric Analysis of Cardiomyocyte Development. 5-AzaC-treated and untreated VSELs were stained for flow cytometric analysis as previously described for characterization of VSELs. Cells were incubated overnight at $4^{\circ} \mathrm{C}$ with primary antibodies (1:200 dilution) against rat-specific cardiac proteins, including cardiac troponin-T (cTnT) (Proteintech) and $\alpha$-sarcomeric actin ( $\alpha$-actin) (Proteintech). After overnight incubation, cells were washed with PBS three 

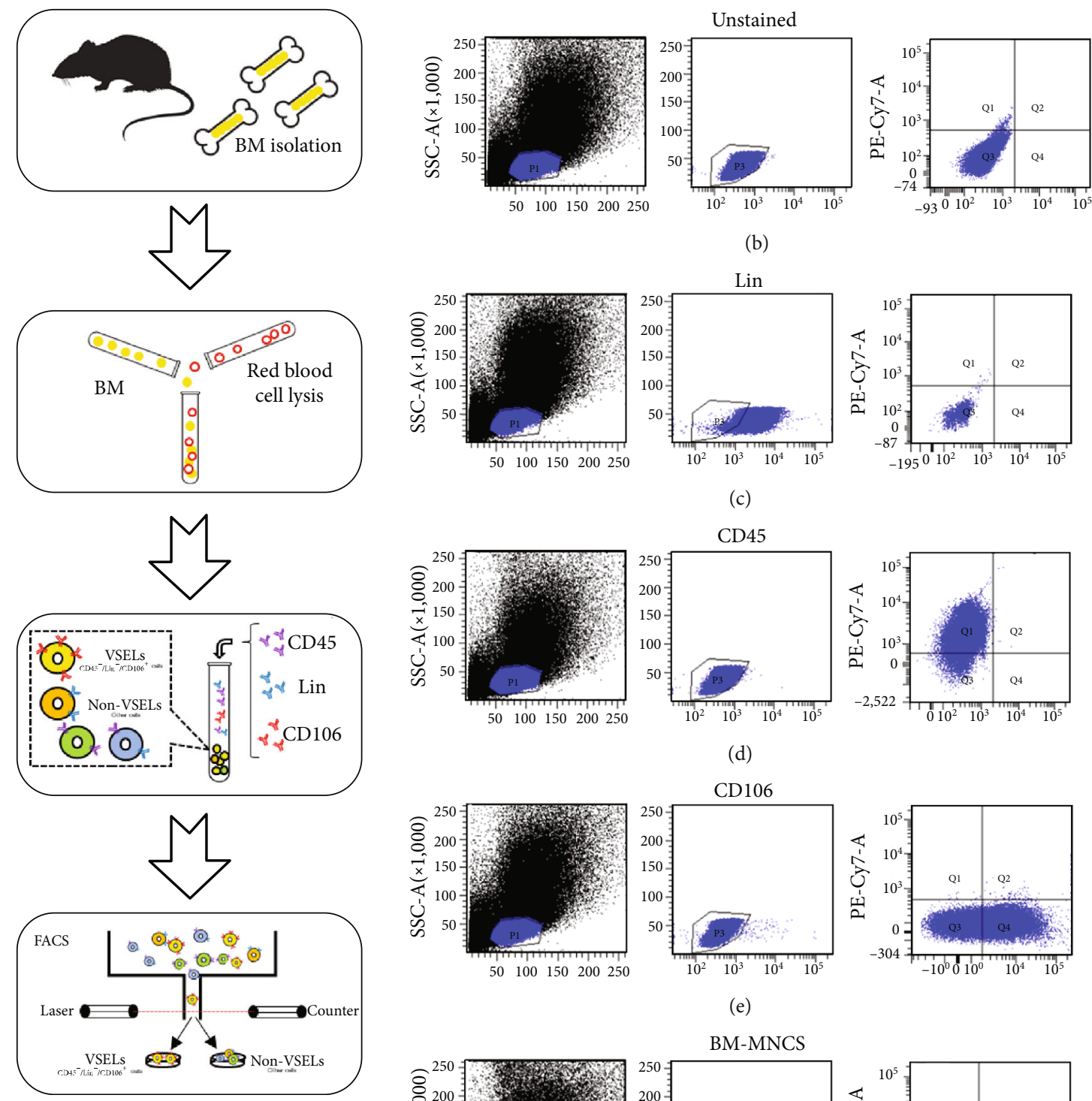

(b)
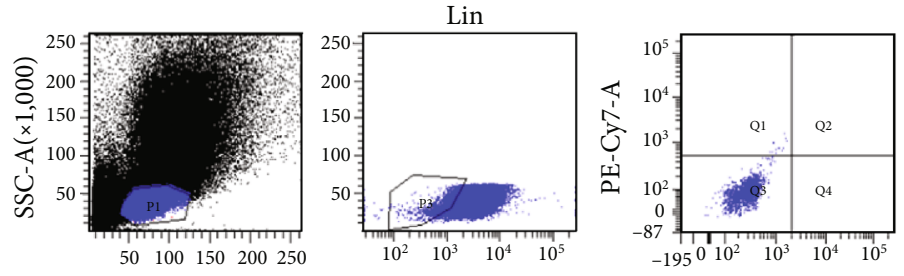

(c)
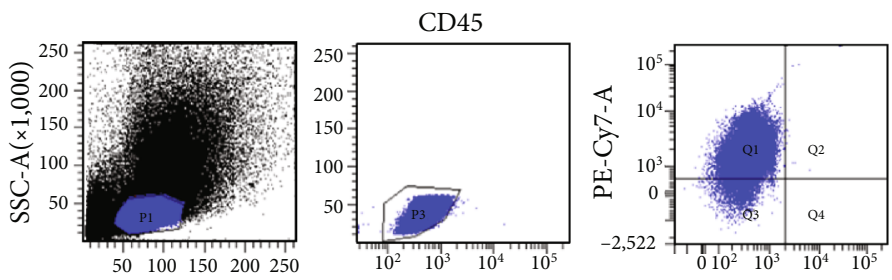

(d)
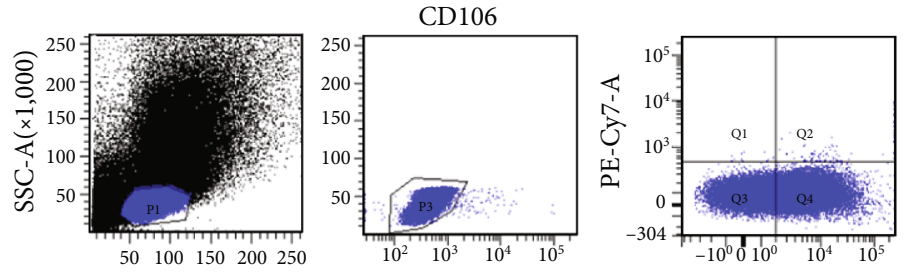

(e)

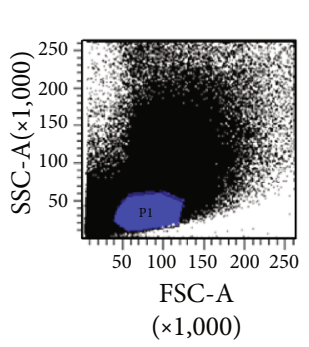

BM-MNCS
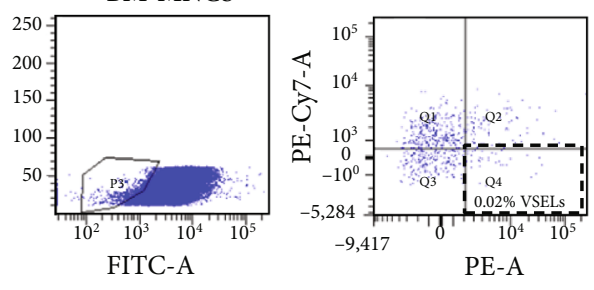

(a)

(f)

FIGURE 1: Rat bone marrow- (BM-) derived very small stem cells (VSELs) were successfully isolated by flow cytometry. (a) The tibia and femur of rats were separated, erythrocytes were lysed, and total BM mononuclear cells were extracted. Fluorophore-conjugated antibodies specific to CD45, CD106, and hematopoietic markers (Lin: TCR $\alpha \beta, \mathrm{CD} 3, \mathrm{CD} 11 \mathrm{~b}$, and CD45RA) were used. Flow cytometric sorting was carried out after incubation. (b) Blank control; (c) Lin control; (d) CD45 control; (e) CD106 control; (f) VSEL sorting.

times for 5 min each, incubated with CY3-conjugated secondary antibody (1:500) for 1 hour at room temperature, and washed five times with PBS. The percentage of red fluorescent protein-positive cells was detected by flow cytometric analysis (BD Biosciences, Franklin Lakes, NJ, USA). Untreated VSELs served as a negative control.

\subsection{RNA Isolation and Reverse Transcription Quantitative} Polymerase Chain Reaction (qPCR) Analysis. Using an
RNeasy Mini Kit (Qiagen, Dusseldorf, Germany), total RNA was extracted from VSELs treated with $5-\mathrm{AzaC}$ at 0 , 7,14 , or 21 days postseeding, according to the manufacturer's instructions. Normal cardiomyocytes were used as the positive control group. cDNA was amplified by PCR using a Qiagen PCR kit according to the manufacturer's instructions. Nanog, Oct-4, Sox2, cTnT, and $\alpha$-actin expression was detected by fluorescent qPCR. The $20 \mu \mathrm{L}$ PCR amplification reaction included $2 \mu \mathrm{L}$ cDNA, $10 \mu \mathrm{L}$ SYBR Taq, $0.8 \mu \mathrm{L}$ 


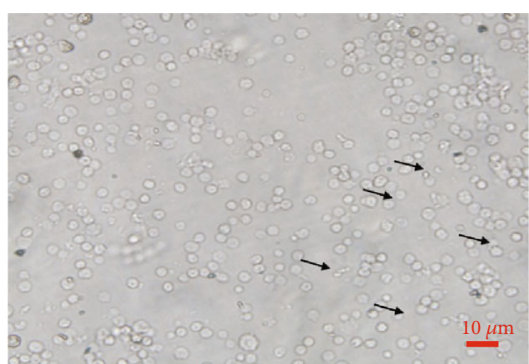

(a)
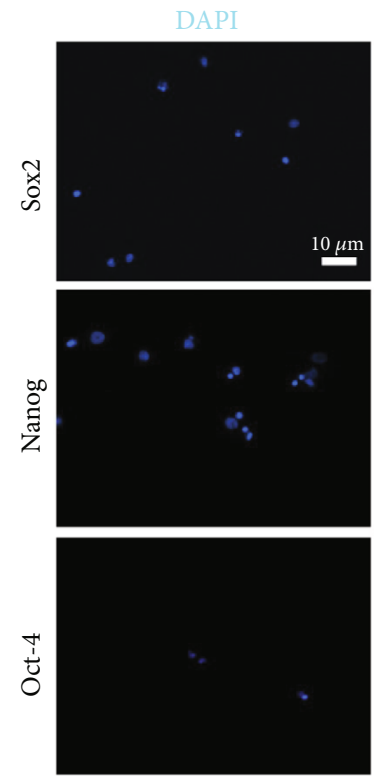

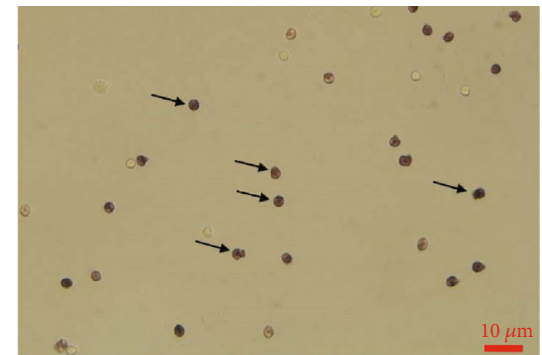

(b)
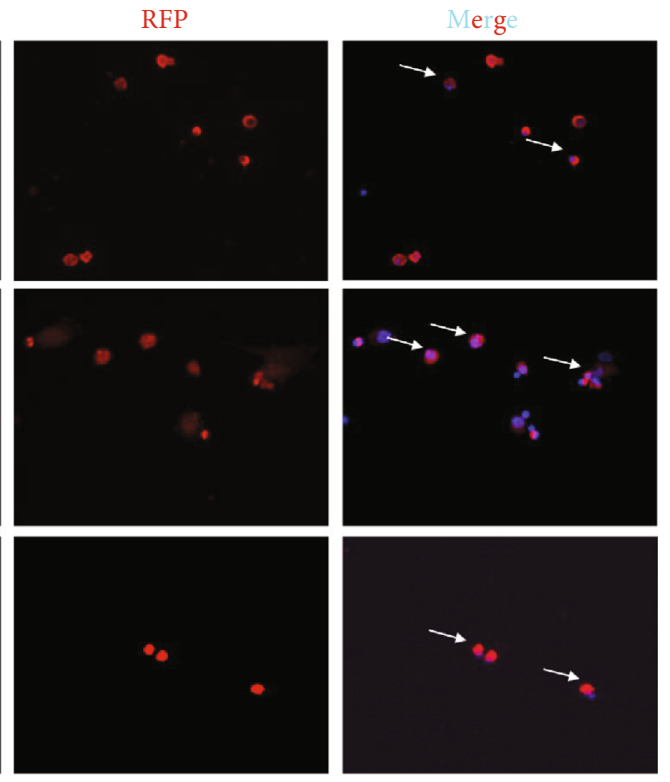

(c)

FIGURE 2: Bone marrow- (BM-) derived very small stem cells (VSELs) retain embryonic stem cell (ESC) characteristics. (a) Rat BM-derived VSELs were obtained by flow cytometric sorting. (b) BM-VSELs were assessed for alkaline phosphatase (AKP) activity by staining for AKP. (c) Expression of Sox2, Oct-4, and Nanog in VSELs was assessed by immunostaining with Sox2-, Oct-4-, and Nanog-specific primary antibodies and a Cy3 secondary antibody.

forward primer, $0.8 \mu \mathrm{L}$ reverse primer, $0.4 \mu \mathrm{L}$ RoxII, and $6 \mu \mathrm{L}$ double-distilled water. Each experimental condition was repeated in triplicate. Relative mRNA quantities were determined using the $2^{-\Delta \Delta \mathrm{CT}}$ method.

2.8. Statistical Analysis. Data obtained were presented as means \pm standard error. Statistical significance was determined using the SPSS 23 statistical program. Variance tests were performed on the data, and GraphPad Prism 7.0 software was used for graphing. The criteria for significance were defined as $* p<0.05, * * p<0.01$, and $* * * p<0.001$.

\section{Results}

3.1. BM-VSELs Retain Characteristics of Embryonic-Derived Stem Cells (ESCs). In this study, VSELs in rat BM were isolated and sorted by flow cytometry. $\mathrm{CD} 45^{-} \mathrm{Lin}^{-} \mathrm{CD} 106^{+}$cells were obtained from BM of adult Wistar rats and defined as VSELs (Figure 1). The majority of VSELs isolated from adult $\mathrm{BM}$ are $<6 \mu \mathrm{m}$, which is larger than peripheral blood platelets, but smaller than erythrocytes (Figure 2(a)). VSELs accounted for $0.020 \pm 0.005 \%$ of the total number of mono- nuclear cells (Figure 1), which is consistent with the results from other laboratories [10].

To determine whether BM-VSELs retained the totipotency of ESCs, the expression of totipotency genes Sox2, Oct-4, and Nanog was detected by immunofluorescence. The results showed that VSELs were positive for Sox2, Oct-4, and Nanog (Figure 2(c)), while cardiomyocytes were negative for these proteins. Additionally, AKP activity test is usually used for undifferentiated embryonic stem cells and primordial germ cells, and in our study, AKP staining test showed positive in VSELs (Figure 2(b)). These results indicated that VSELs were successfully isolated from rat BM and retained biological characteristics similar to ESCs.

3.2. 5-AzaC Induces Cardiomyocyte-Like Morphology Changes in BM-VSELs. To investigate the feasibility of 5AzaC to induce VSEL differentiation into cardiomyocytes, VSELs were treated with 5-AzaC at multiple concentrations and assessed for cardiomyocyte morphology. Without 5AzaC treatment, there were no obvious morphological changes in the untreated control group (Figure 3(b)). VSELs 


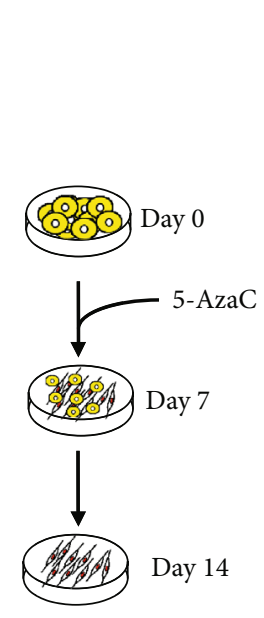

(a)
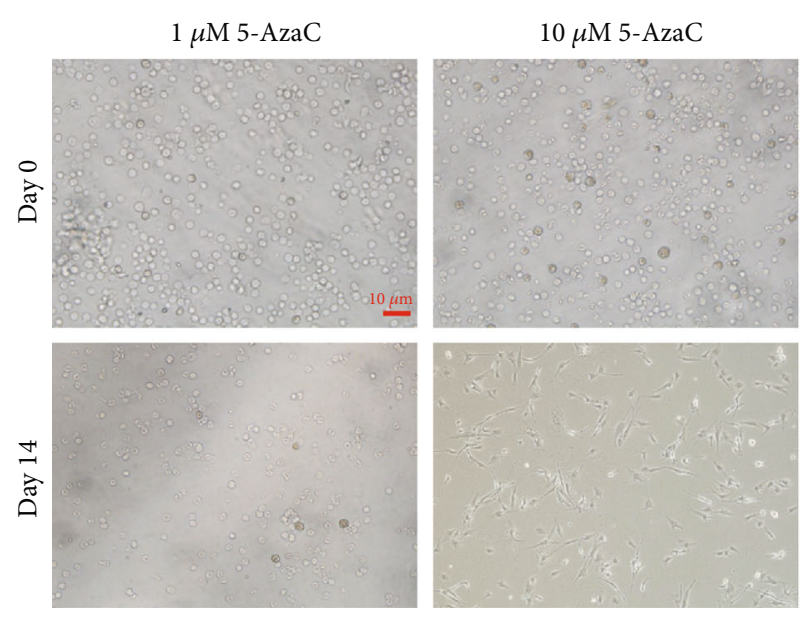

(b)

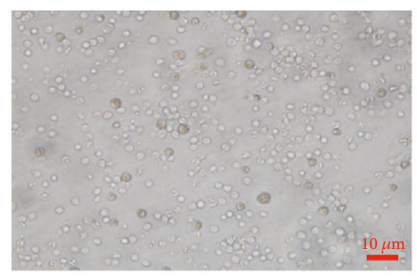

Day 0

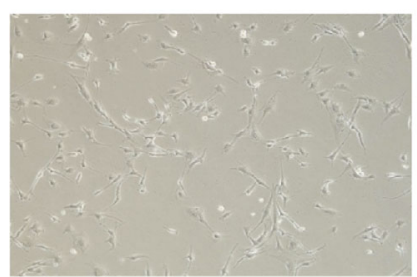

Day 14

$\neg 30 \mathrm{mM}$

$\rightarrow$ 5-AzaC-

(c)
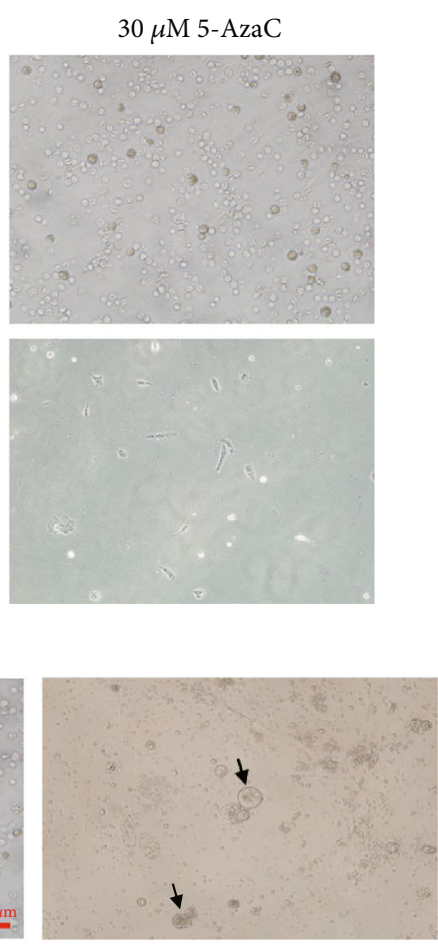

Day 7

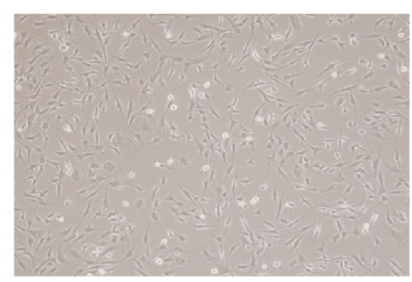

Day 21

FIGURE 3: 5-AzaC induces cardiomyocyte-like morphology in BM-VSELs. (a) 5-AzaC-induced VSELs showed morphological changes at day 7, and the population of cardiomyocytes peaked at day 14. (b) Growth of VSELs treated with different concentrations of 5-AzaC (1 $\mu \mathrm{M}$, $10 \mu \mathrm{M}$, and $30 \mu \mathrm{M}$ ). (c) MTT assay indicated VSEL growth induced by different concentrations of 5-AzaC over time. (d) Morphological changes in BM-VSELs after treatment with $10 \mu \mathrm{M}$ 5-AzaC. The arrow indicates that VSELs developed embryoid body-like structures after 5-AzaC treatment.

treated with $1 \mu \mathrm{M}$ 5-AzaC became slightly more ovular, but did not form the typical cardiomyocyte morphology. Additionally, VSELs treated with $30 \mu \mathrm{M}$ 5-AzaC showed abnormal cell morphology and enhanced cell death. After exposure to $10 \mu \mathrm{M}$ 5-AzaC for 24 hours, VSELs were cultured in normal medium. Embryoid body- (EB-) like structures developed in VSELs on day 4 posttreatment. After 5-7 days posttreatment in culture, the cytoplasm gradually stretched to form rod-like structures (Figure 3(d)). Myotube-like morphology was observed in the cultured cells after 14 days posttreatment. At day 21, there was little change in morphology from day 14, and the existing cells began to undergo apoptosis (Figures 3(a) and 3(d)). Cell viability analysis by MTT assay at days $0,1,7,14$, and 21 post-5-AzaC treatment revealed that
$10 \mu \mathrm{M}$ yielded the highest number of viable cells. Therefore, $10 \mu \mathrm{M}$ is the appropriate concentration of $5-\mathrm{AzaC}$ to be used in BM-VSEL treatment (Figure 3(c)).

\subsection{5-AzaC Induces BM-VSEL Differentiation into} Cardiomyocytes. To evaluate the differentiation of BMVSELs into cardiomyocytes, the expression of specific proteins known to be important for cardiomyocyte formation and function was investigated. After 14 days in culture, immunofluorescence staining indicated that BM-VSELs were strongly positive for cardiomyocyte markers, including cTnT and $\alpha$ actin (Figure 4(a)). Conversely, no positive cells were detected in the untreated control group. Furthermore, the flow cytometric analysis revealed that the number of cTnT- and $\alpha$ - 


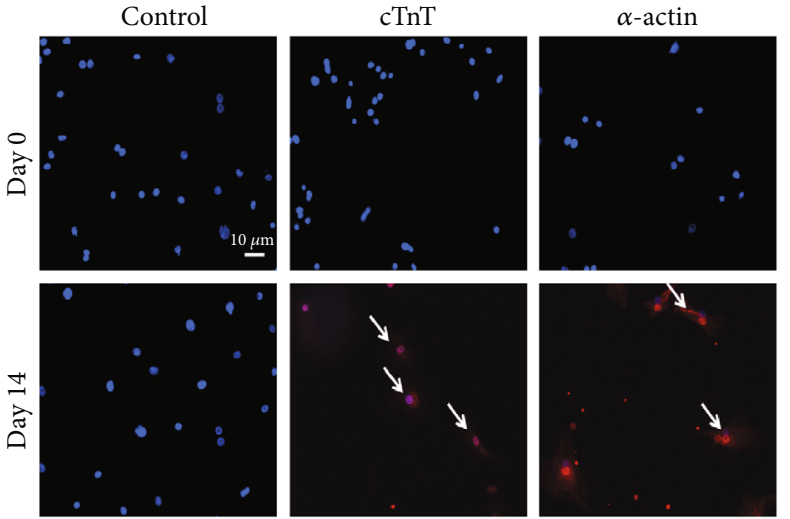

(a)
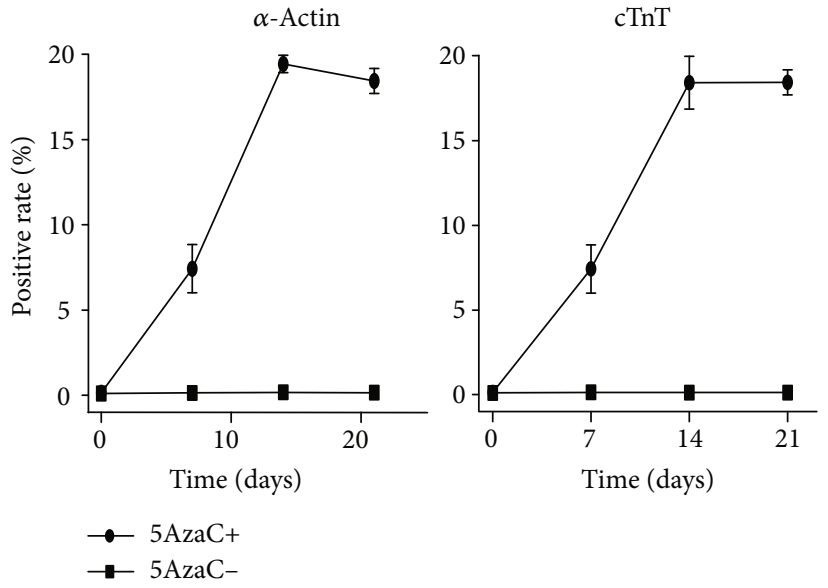

(b)

Figure 4: 5-AzaC treatment increased levels of cardiac troponin-T (cTnT) and $\alpha$-sarcomeric actin ( $\alpha$-actin) in BM-VSELs. (a) Immunostaining for $\mathrm{cTnT}$ and $\alpha$-actin in cardiomyocytes derived from 5-AzaC-induced differentiation of VSELs. The white arrow indicates positive cells. (b) Flow cytometric analysis demonstrated the percentages of cTnT- and $\alpha$-actin-positive cells on different days.

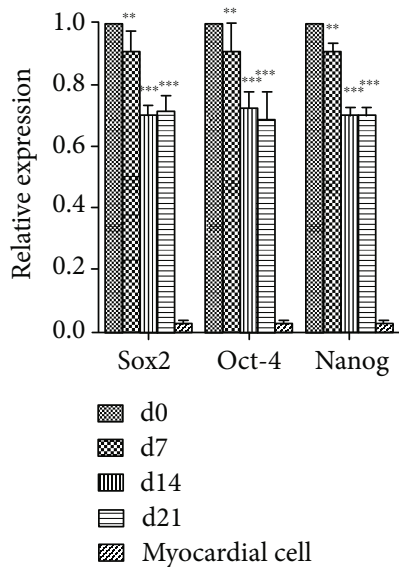

(a)

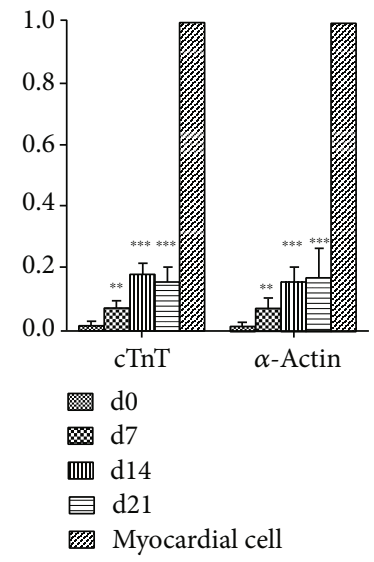

(b)

Figure 5: Gene expression levels in BM-VSELs differentiated into cardiomyocytes after 5-AzaC treatment. Expression of (a) Sox2, Oct-4, and Nanog and (b) cTnT and $\alpha$-actin was detected in 5-AzaC-treated cells via qRT-PCR. The gene expression levels of cardiomyocytes and pluripotent stem cell genes were normalized with GAPDH and compared to the relative control group and cardiomyocytes $(n=3$, level of significance $p<0.05 ; * p<0.05 ; * * p<0.01 ; * * * p<0.001)$.

actin-positive cells began to rise at day 7 and peaked at day 14 posttreatment, showing a significant increase from day 0 $(p<0.001)$. BM-VSEL cTnT- and $\alpha$-actin-positive expression rates reached $18.41 \pm 1.51 \%$ and $19.43 \pm 0.51 \%$, respectively. However, there was no significant difference between days 14 and $21 \quad(p>0.05)$ (Figure 4(b)). These changes suggest that after exposure to 5-AzaC, the differentiation efficiency of BM-VSELs into cardiac cells was approximately $20 \%$.

3.4. 5-AzaC Treatment Reduces Expression of Pluripotent Stem Cell Genes in BM-VSELs. To evaluate changes in gene expression in VSELs differentiated into cardiomyocytes, we assessed expression of cardiomyocyte-specific and stem cell-specific genes by qPCR. After treatment with $10 \mu \mathrm{M} 5$ AzaC, VSELs continued to proliferate and differentiate. The expression levels of cardiomyocyte-specific transcripts cTnT and $\alpha$-actin gradually increased at day 7 , with significant differences between day 0 and days 7, 14, and $21 \quad(p<0.05)$ (Figure 5(b)). The expression levels of stem cell-associated genes Sox2, Oct-4, and Nanog gradually decreased in 5AzaC-treated VSELs $(p<0.05)$ (Figure 5(a)). The decrease in pluripotent stem cell gene expression and the increase in myocardium-specific gene expression in VSELs after 5AzaC treatment indicated that VSELs successfully differentiated into cardiomyocytes. 


\section{Discussion}

Compared with implantable electronic pacemakers, biological pacemakers are more suitable for treating chronic arrhythmia and pathological sinus syndrome [1]. Moreover, large-animal studies have shown that biological pacing is an effective way to reconstruct the pacing function of sinoatrial node cells [11]. This study was conducted to investigate the ability of BMVSELs to differentiate into cardiomyocytes after treatment with 5-AzaC in vitro to provide sufficient numbers of wellconditioned seed cells for cardiomyocyte differentiation.

ESCs [12], induced pluripotent stem cells (iPSs) [13], and adult stem cells [14-16] are the most common seed cells used for biological pacing. Due to ethical concerns, rejection, tumor-forming tendency, and safety, there are still limitations to their clinical application [17]. Therefore, new biological pacemaker candidate cell types are needed.

VSELs represent a unique and rare population of adult stem cells sharing several structural, genetic, biochemical, and functional properties with ESCs [2]. They have also been identified in several adult murine and human tissues, including ovaries and testicles [4]. VSELs offer several major advantages over currently available seed cells. Given their pluripotent nature and ability to differentiate into cardiomyocytes or endothelial cells, VSELs are particularly well suited for cellular-replacement therapy. The expression of various angiogenic and protective factors in VSELs also renders them suitable for myocardial repair via paracrine actions. Furthermore, unlike the currently available pluripotent cells (ESCs and iPSs), VSELs do not form tumors during extended follow-up studies. Finally, since VSELs can be isolated from adult tissues, the use of autologous VSELs not only avoids rejection and other potential immune risks but also avoids ethical issues [10]. Although VSELs have been isolated from mouse and human tissue for more than a decade, VSELs were not isolated from rat bone marrow until 2016 [4]. Compared with mouse and humans, isolating VSELs from rats is easier and less ethically controversial. In our work, VSELs with high expression of Sox2, Oct-4, and Nanog were successfully isolated from rat BM according to the experimental method of Labedz-Maslowska et al. [4]. VSELs accounted for $0.01-0.02 \%$ of the total number of nucleated cells, which was consistent with previous reports $[1,4,18]$.

The study by Kassmer et al. [19] confirmed that mousederived VSELs can differentiate into lung epithelial cells (endoderm) in vivo and Oct-4-positive VSELs have the potential for transdermal differentiation. Mouse BM-VSELs can be differentiated into cardiomyocytes (mesoderm), neurons, astrocytes, oligodendrocytes (ectoderm), and islet cells (endoderm) [20]. Our study may be the first attempt to induce differentiation of rat BM-VSELs into cardiomyocytes.

At present, there are many ways to induce the differentiation of stem cells into cardiomyocytes in vitro, including chemical inducers, coculture, conditioned medium of cardiomyocytes [21], and bionic electrical stimulation [22]. However, the chemical inducer and DNA methyltransferase inhibitor 5-AzaC is widely used due to low cost, easy operation, and dosage control. 5-AzaC has been successfully used in the cardiac differentiation of mesenchymal stem cells
(MSCs) and human ESCs $[23,24]$. The mechanism of 5AzaC may be related to CpG base-pair demethylation and regulation of early myocardial transcription factors [25-28]. In this study, cultured BM-VSELs were treated with different concentrations of 5-AzaC for 24 hours. The $10 \mu \mathrm{M} \mathrm{5-AzaC}$ conditions resulted in differentiation of VSELs into cardiomyocyte with fewer apoptotic cells. The concentration of 5$\mathrm{AzaC}$ we used was consistent with the concentration of cardiomyocyte growth induced by Antonitsis et al. in adult bone marrow MSCs [25].

EBs are used as an in vitro model for evaluating early extraembryonic tissue formation and differentiation processes. After 48 hours of treatment with $5 \mu \mathrm{M}$ 5-AzaC, Diomede et al. [29] found that human gingival MSCs (hGMSCs) were organized as round 3D structures (EBshGMSCs). In our study, EBs appeared at day 4, later than the hGMSCs induced by Diomede et al. using 5-AzaC, which may be due to the fact that VSELs are a group of inactive heterogeneous cells [30]. After treatment with 5-AzaC, VSELs gradually form muscular tubular structures. Rod-like morphological changes may be related to the expression of proteins that maintain the cytoskeleton.

The increased expression of myocardial-specific proteins and the reduced expression level of pluripotent stem cell genes indicate the transformation of VSELs to cardiomyocytes. It is speculated that genes controlling the differentiation of VSELs into cardiomyocytes are activated and that VSELs successfully differentiate into cardiomyocytes. This may be because 5 -AzaC can induce ultrastructural changes in cells, thereby activating key transcription factors and differentiating cells into cardiomyocytes [25]. We found that the number of cTnT- and $\alpha$-actin-positive cells was approximately $20 \%$ at day 14 , which is similar to 5 -AzaC-induced MSC differentiation efficiency [25].

\section{Conclusion}

This study describes the successful isolation of VSELs containing multidirectional differentiation potential from rat BM tissue. Furthermore, the VSELs differentiated into cardiomyocytes through 5-AzaC treatment. These results have important implications for regenerative medicine related to cell therapy of chronic arrhythmias.

\section{Data Availability}

The data used to support the findings of this study are available from the corresponding author upon request.

\section{Conflicts of Interest}

The authors declare no potential conflicts of interest.

\section{Acknowledgments}

We gratefully acknowledge Dr. Bichun Li for the help and support with performing these experiments. This trial was financed by the Science and Technology Department of Jiangsu Province (funding code BL2013022) . 


\section{References}

[1] C. G. Burns and C. E. Burns, "Canonical Wnt signaling sets the pace,” Developmental Cell, vol. 50, no. 6, pp. 675-676, 2019.

[2] M. Kucia, R. Reca, F. R. Campbell et al., "A population of very small embryonic-like (VSEL) CXCR $4^{+}$SSEA $-1^{+}$Oct- $4^{+}$stem cells identified in adult bone marrow," Leukemia, vol. 20, no. 5, pp. 857-869, 2006.

[3] E. R. Burnight, M. Gupta, L. A. Wiley et al., "Using CRISPRCas 9 to generate gene-corrected autologous iPSCs for the treatment of inherited retinal degeneration," Molecular Ther$a p y$, vol. 25, no. 9, pp. 1999-2013, 2013.

[4] A. Labedz-Maslowska, E. Kamycka, S. Bobis-Wozowicz, Z. Madeja, and E. K. Zuba-Surma, "Identification of new rat bone marrow-derived population of very small stem cell with Oct-4A and Nanog expression by flow cytometric platforms," Stem Cells International, vol. 2016, Article ID 5069857, 14 pages, 2016.

[5] M. Kucia, M. Halasa, M. Wysoczynski et al., "Morphological and molecular characterization of novel population of CXCR4+ SSEA-4+ Oct-4+ very small embryonic-like cells purified from human cord blood: preliminary report," Leukemia, vol. 21, no. 2, pp. 297-303, 2007.

[6] D. Bhartiya and K. James, "Very small embryonic-like stem cells (VSELs) in adult mouse uterine perimetrium and myometrium," Journal of Ovarian Research, vol. 10, no. 1, p. 29, 2017.

[7] D. M. Smadja, "Bone marrow very small embryonic-like stem cells: new generation of autologous cell therapy soon ready for prime time?," Stem Cell Reviews and Reports, vol. 13, no. 2, pp. 198-201, 2017.

[8] E. K. Zuba-Surma, W. Wojakowski, M. Z. Ratajczak, and B. Dawn, "Very small embryonic-like stem cells: biology and therapeutic potential for heart repair," Antioxidants \& Redox Signaling, vol. 15, no. 7, pp. 1821-1834, 2011.

[9] K. Govarthanan, P. K. Gupta, D. Ramasamy, P. Kumar, S. Mahadevan, and R. S. Verma, "DNA methylation microarray uncovers a permissive methylome for cardiomyocyte differentiation in human mesenchymal stem cells," Genomics, vol. 112, no. 2, pp. 1384-1395, 2020.

[10] M. Z. Ratajczak, E. K. Zuba-Surma, M. Wysoczynski, J. Ratajczak, and M. Kucia, "Very small embryonic-like stem cells: characterization, developmental origin, and biological significance," Experimental Hematology, vol. 36, no. 6, pp. 742-751, 2008.

[11] N. Nishii, "Arrhythmia management after device removal," Journal of arrhythmia, vol. 32, no. 4, pp. 287-292, 2016.

[12] H. C. Cho, Y. Kashiwakura, and E. Marbán, "Creation of a biological pacemaker by cell fusion," Circulation Research, vol. 100, no. 8, pp. 1112-1115, 2007.

[13] K. H. So, Y. J. Han, H. Y. Park et al., "Generation of functional cardiomyocytes from mouse induced pluripotent stem cells," International Journal of Cardiology, vol. 153, no. 3, pp. 277285, 2011.

[14] C. Jun, Z. Zhihui, W. Lu et al., "Canine bone marrow mesenchymal stromal cells with lentiviral mHCN4 gene transfer create cardiac pacemakers," Cytotherapy, vol. 14, no. 5, pp. 529-539, 2012.

[15] X. J. Yang, Y. F. Zhou, H. X. Li, L. H. Han, and W. P. Jiang, "Mesenchymal stem cells as a gene delivery system to create biological pacemaker cells in vitro," The Journal of International Medical Research, vol. 36, no. 5, pp. 1049-1055, 2008.
[16] V. Planat-Bénard, C. Menard, M. André et al., "Spontaneous cardiomyocyte differentiation from adipose tissue stroma cells," Circulation Research, vol. 94, no. 2, pp. 223-229, 2004.

[17] G. Koifman, Y. Shetzer, S. Eizenberger et al., “A mutant p53dependent embryonic stem cell gene signature is associated with augmented tumorigenesis of stem cells," Cancer Research, vol. 78, no. 20, pp. 5833-5847, 2018.

[18] A. Shaikh, P. Nagvenkar, P. Pethe, I. Hinduja, and D. Bhartiya, "Molecular and phenotypic characterization of CD133 and SSEA4 enriched very small embryonic-like stem cells in human cord blood," Leukemia, vol. 29, no. 9, pp. 1909-1917, 2015.

[19] S. H. Kassmer, H. Jin, P. X. Zhang et al., "Very small embryonic-like stem cells from the murine bone marrow differentiate into epithelial cells of the lung," Stem Cells, vol. 31, no. 12, pp. 2759-2766, 2013.

[20] M. Kucia, W. Wu, and M. Z. Ratajczak, "Bone marrow-derived very small embryonic-like stem cells: their developmental origin and biological significance," Developmental dynamics: an official publication of the American Association of Anatomists, vol. 236, no. 12, pp. 3309-3320, 2007.

[21] S. Rangappa, J. W. C. Entwistle, A. S. Wechsler, and J. Y. Kresh, "Cardiomyocyte-mediated contact programs human mesenchymal stem cells to express cardiogenic phenotype," The Journal of Thoracic and Cardiovascular Surgery, vol. 126, no. 1, pp. 124-132, 2003.

[22] E. Serena, E. Figallo, N. Tandon et al., "Electrical stimulation of human embryonic stem cells: cardiac differentiation and the generation of reactive oxygen species," Experimental Cell Research, vol. 315, no. 20, pp. 3611-3619, 2009.

[23] L. Cimmino, "Methylation maintains HSC division fate," Proceedings of the National Academy of Sciences of the United States of America, vol. 114, no. 2, pp. 192-194, 2017.

[24] D. Abbey and P. B. Seshagiri, "Aza-induced cardiomyocyte differentiation of P19 EC-cells by epigenetic co-regulation and ERK signaling," Gene, vol. 526, no. 2, pp. 364-373, 2013.

[25] P. Antonitsis, E. Ioannidou-Papagiannaki, A. Kaidoglou, and C. Papakonstantinou, "In vitro cardiomyogenic differentiation of adult human bone marrow mesenchymal stem cells. The role of 5-azacytidine," Interactive CardioVascular and Thoracic Surgery, vol. 6, no. 5, pp. 593-597, 2007.

[26] F. Larsen, G. Gundersen, R. Lopez, and H. Prydz, "CpG islands as gene markers in the human genome," Genomics, vol. 13, no. 4, pp. 1095-1107, 1992.

[27] Z.-B. Ruan, L. Zhu, Y.-G. Yin, and G.-C. Chen, "The mechanism underlying the differentiation of human umbilical cordderived me-senchymal stem cells into myocardial cells induced by 5-azacytidine," Indian Journal of Medical Sciences, vol. 64, no. 9, pp. 402-407, 2010.

[28] B. P. Enright, C. Kubota, X. Yang, and X. C. Tian, "Epigenetic characteristics and development of embryos cloned from donor cells treated by trichostatin A or 5-aza-2'-deoxycytidine," Biology of Reproduction, vol. 69, no. 3, pp. 896-901, 2003.

[29] F. Diomede, N. Zini, and J. Pizzicannella, "5-Aza exposure improves reprogramming process through embryoid body formation in human gingival stem cells," Frontiers in Genetics, vol. 9, p. 419, 2018.

[30] A. Heider, R. Danova-Alt, D. Egger, M. Cross, and R. Alt, "Murine and human very small embryonic-like cells: a perspective," Cytometry Part A, vol. 83A, no. 1, pp. 72-75, 2013. 